\title{
How Facilitation May Interfere with Ecological Speciation
}

\author{
P. Liancourt, ${ }^{1,2}$ P. Choler, ${ }^{3,4}$ N. Gross, ${ }^{5,6}$ X. Thibert-Plante, ${ }^{7}$ and K. Tielbörger ${ }^{2}$ \\ ${ }^{1}$ Department of Biology, University of Pennsylvania, 322 Leidy Labs, Philadelphia, PA 19104, USA \\ ${ }^{2}$ Department of Plant Ecology, University of Tübingen, Auf der Morgenstelle 3, 72072 Tübingen, Germany \\ ${ }^{3}$ Laboratoire d'Ecologie Alpine, CNRS UMR 5553, Université de Grenoble, BP 53, 38041 Grenoble, France \\ ${ }^{4}$ Station Alpine J. Fourier, CNRS UMS 2925, Université de Grenoble, 38041 Grenoble, France \\ ${ }^{5}$ INRA, USC Agripop (CEBC-CNRS), 79360 Beauvoir sur Niort, France \\ ${ }^{6}$ CEBC-CNRS (UPR 1934), 79360 Beauvoir sur Niort, France \\ ${ }^{7}$ National Institute for Mathematical and Biological Synthesis (NIMBioS), University of Tennessee, 1534 White Avenue, Suite 400, \\ Knoxville, TN 37996, USA
}

Correspondence should be addressed to P. Liancourt, plia@sas.upenn.edu

Received 13 July 2011; Revised 15 November 2011; Accepted 10 December 2011

Academic Editor: Rui Faria

Copyright () 2012 P. Liancourt et al. This is an open access article distributed under the Creative Commons Attribution License, which permits unrestricted use, distribution, and reproduction in any medium, provided the original work is properly cited.

\begin{abstract}
Compared to the vast literature linking competitive interactions and speciation, attempts to understand the role of facilitation for evolutionary diversification remain scarce. Yet, community ecologists now recognize the importance of positive interactions within plant communities. Here, we examine how facilitation may interfere with the mechanisms of ecological speciation. We argue that facilitation is likely to (1) maintain gene flow among incipient species by enabling cooccurrence of adapted and maladapted forms in marginal habitats and (2) increase fitness of introgressed forms and limit reinforcement in secondary contact zones. Alternatively, we present how facilitation may favour colonization of marginal habitats and thus enhance local adaptation and ecological speciation. Therefore, facilitation may impede or pave the way for ecological speciation. Using a simple spatially and genetically explicit modelling framework, we illustrate and propose some first testable ideas about how, when, and where facilitation may act as a cohesive force for ecological speciation. These hypotheses and the modelling framework proposed should stimulate further empirical and theoretical research examining the role of both competitive and positive interactions in the formation of incipient species.
\end{abstract}

\section{Introduction}

Over the last 20 years, there has been increasing recognition that besides competition, facilitation, that is, the amelioration of biotic and/or abiotic conditions by neighboring organisms, is a key driver controlling assembly and dynamics of communities ([1], see [2] for recent reviews). The impact of facilitation in a community ecology context has been now widely accepted, but its evolutionary consequences have been rarely explored $[2,3]$. There also has been an increasing awareness that positive and negative interactions usually cooccur in nature, and recent works have turned to examine conditions under which the outcome of biotic interactions is positive, negative, or neutral [4-6]. Yet, while there is a long-standing literature linking competitive interactions and speciation [7-10], the role of facilitation for evolutionary diversification has been largely overlooked. Very recently, a few empirical studies have attempted to bridge this gap [11$13]$.

The theoretical study of positive interactions in an evolutionary context has an old history (e.g., [14]), but these models only consider mutualism, a two-species interaction that benefits both partners [15]. However, mutualism is characterized by balanced reciprocal benefits for species involved in a one-to-one relationship, while facilitation encompasses a larger spectrum of positive interactions between organisms $[2,16]$. Namely, it commonly includes one-to-many interactions where one organism ameliorates habitat conditions for several beneficiary organisms and many-to-one interactions where an organism benefits from diffuse facilitation by neighbors. For example, facilitation between plants occurs when neighboring vegetation or a nurse plant (more 
generally called a benefactor) ameliorates environmental conditions directly, through the provision of additional resources or shelter from extreme conditions (e.g., high or low temperatures), or indirectly through protection from herbivores or via attraction of pollinators [16]. Since facilitation generates local variation in environmental conditions, it should also affect the manner in which organisms respond and adapt to their environment. However, it is important to realize that facilitation differs intrinsically from microhabitat amelioration due to abiotic components of the system (e.g., rock, ponds, or nutrient patches) because the source of environmental heterogeneity is a living organism. For instance, the distribution of favourable microhabitats may change at the time scale of ecological responses because of the population dynamics of benefactor species [17]. Additionally, the net effect of benefactors can shift from facilitation to competition due to interannual resource dynamics (e.g., change in rainfall pulse [18]).

The growing interest for eco-evolutionary dynamics, that is, the interplay between ecological and evolutionary processes [19-22], demonstrates the need to study both the evolutionary consequences of ecological processes and the effect of evolutionary processes on ecological responses. In a recent study, Michalet et al. [17] provided one specific aspect of the problem from the benefactor's point of view. Namely, they could show that ecotypic variation in benefactor traits may modify the manner in which they affect understory species. Viceversa, the understory also affected fitness of the benefactor. Here, we tackle this problem by looking at the other side of this coin and from a more general perspective, that is, the evolutionary consequences of facilitation on diversification of beneficiaries.

We aim at complementing the vast literature on competitive effects on evolutionary diversification by a conceptual model addressing the role of facilitation on distribution range, local adaptation, and genetic structure of beneficiary species. In particular, we discuss the likely effect of facilitation on microevolutionary processes and ultimately on ecological speciation. Ecological speciation can be defined as a particular type of speciation, where reproductive isolation evolves between populations as a result of ecologically based divergent selection [23-25]. In this context, the interplay between biotic and abiotic factors is of paramount importance and, not surprisingly, the attempts to link ecological and evolutionary processes are the most tantalizing $[3,17$, 26].

We do not present an exhaustive list of all potential evolutionary consequences of facilitation but instead propose an alternative to traditional views on the role of biotic interactions for evolutionary processes. Our overarching hypothesis is that, contrary to competition, facilitation may impede ecological speciation by maintaining gene flow between incipient species and preventing niche partitioning. Alternatively, we also discuss under which more specific circumstances facilitation could act as a stepping stone to promote ecological speciation. First, we highlight the role of facilitation in the context of niche theory. Secondly, we present how facilitation is likely to interfere with microevolutionary processes and propose a modeling framework to illustrate our arguments (Box 1). Lastly, we call for a new body of empirical and theoretical works to test the presented hypotheses (Box 2). The ideas and hypotheses developed in this paper are biased towards the plant kingdom (as is the facilitation literature in general, e.g., $[2,16]$ ) but should apply equally to other kingdoms. For example, there is now some evidence showing the importance of facilitation for a wide range of organisms such as terrestrial animals [27], marine invertebrates [28], fungi [29], bacteria [3], and viruses [30].

\section{Facilitation and Niche Theory, Moving from Species to Genotypes}

Facilitation is likely to be as common as competition along environmental gradients [2]. It is generally hypothesized to increase in importance as environmental severity increases ([1], but see [31]) and is likely to enhance species diversity in harsh habitats [31-34]. It is now recognized that facilitation can expand the distribution range of a species at its harsher end, or marginal habitat [35], whereas competition generally reduces it at the favorable end of the environmental gradient $[4,5,36,37]$. By widening the distribution range of certain species not adapted to stressful conditions, facilitation allows them to persist with subordinate $[4,5,33,34,36]$ or even dominant status in environments, where without facilitation they would not be able to persist [37]. Therefore, within a particular community, the ability of a species to be facilitated is strongly linked with its own niche $[4,5,37]$ in that only species deviated from their niche optima are likely to be facilitated. More specifically, competitive species dominating favorable environments are more affected by facilitation as stress increases than stress-tolerant species adapted to harsher ecological conditions. Conversely, stresstolerant species are competitively excluded as environmental stress decreases [31] due to a trade-off between competitive ability and stress-tolerance (see [5]).

Here, we apply these concepts developed on a species level to variation among incipient species. Namely, we propose to establish a missing link between facilitation as a force structuring communities and one affecting population structure. Due to the fact that different genotypes within a species may differ in their resource use and stress tolerance $[38,39]$, they can be characterized by contrasting ecological tolerances and different optima. For example, local adaptation along environmental gradients [40] will lead to ecotypespecific "realized niches." Note that this concept is commonly incorporated in models of resource competition (e.g., [41, $42])$. On the one hand, genotypes exhibiting traits linked to stress tolerance should be selected for, and thus be more common at the margin of the species' realized niche (harsh conditions). On the other hand, genotypes enabling larger competitive response ability and low stress tolerance should dominate in populations in the center of the species niche, that is, more in favorable environments $[43,44]$.

Any ecological process that has the potential to significantly impact gene flow in marginal habitats may play a key role in ecological speciation $[45,46]$. In that context, dispersal-related processes have been well investigated [43]. Here, we argue that the likelihood that core genotypes can 

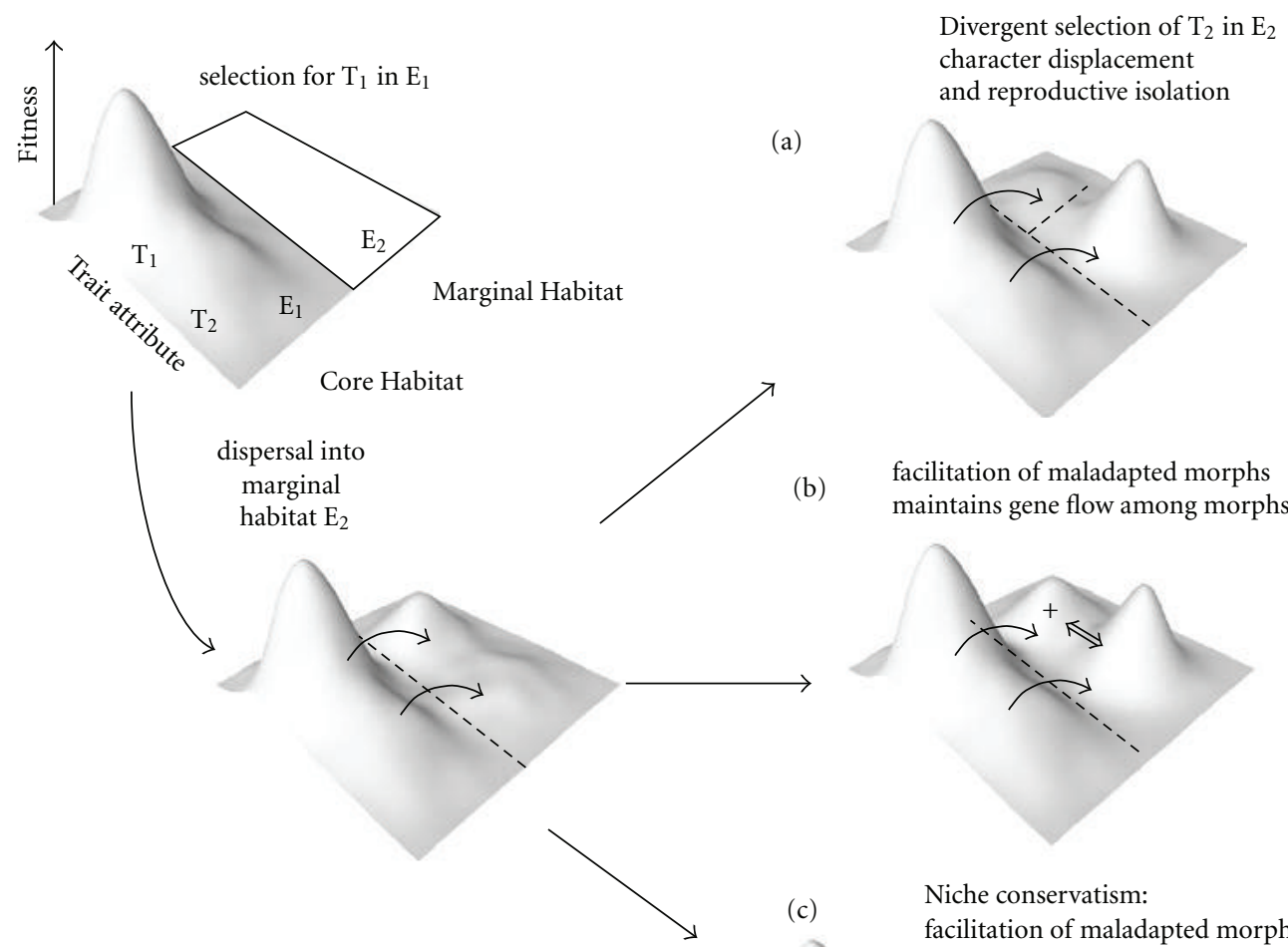

(b)

facilitation of maladapted morphs maintains gene flow among morphs

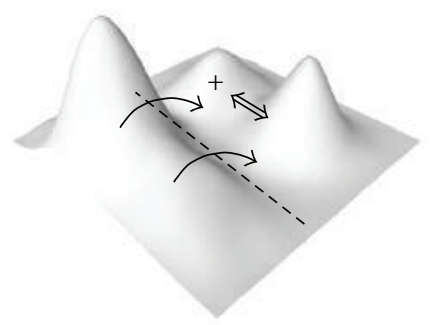

(c)

Niche conservatism:

facilitation of maladapted morphs

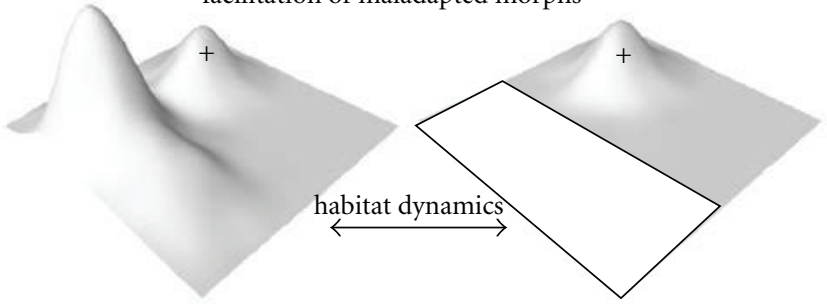

FIGURE 1: How facilitation may interfere with ecological speciation and niche conservatism. These 3D plots sketch ongoing disruptive selection and trait divergence of incipient species. The vertical axis represents the relative fitness of genotypes in a trait-ecological space. We assumed that ecologically marginal conditions (E2) also correspond to peripheral areas. Solid arrows are for gene flow including dispersal of propagules, individuals, and sexual reproduction. Dotted lines denote reproductive isolation. A common starting point is immigration of genotypes from the favorable part of the gradient to marginal/peripheral habitats. (a) Diversifying selection without facilitation, and reproductive isolation that eventually arises as a by-product of trait divergence. (b) Maladapted forms persist in marginal habitats because of facilitation and a maintained gene flow among genotypes prevents ecological speciation. (c) Facilitation allows maladapted forms to persist in a changing environment, that is, niche conservatism.

persist in a species' ecological margin without facilitation is particularly low. In the likely scenario of net dispersal from core (source) to marginal habitats (sinks) [43], facilitation may enhance the establishment of core genotypes in habitats where they otherwise would not be able to persist. This will have crucial consequences for adaptation to marginal habitats, and in turn for species diversification along environmental gradients, which we explain below.

\section{Consequence of Facilitation for Gene Flow and Genetic Structure of Beneficiaries}

A first mechanism by which facilitation may impact adaptive diversification is in enabling maladapted genotypes/forms to persist and reproduce outside of their niche optima. Figure 1 depicts two habitat types with contrasting environmental conditions (core and marginal), where different genotypes are distributed. Genotypes are supposed to differ by a key trait under selective pressure (e.g., a stress-tolerance-related trait). Local adaptation to marginal habitats is possible if gene flow from core habitats is limited due to pre- or postzygotic barriers ([47], but see [48]). For example, ecological filtering is a prezygotic barrier that will select genotypes best adapted to the local conditions and will reduce viability of the immigrants [49]. Postzygotic barriers may include reduced fitness of hybrids related to stress intolerance or to competitive displacement by more adapted forms $[50,51]$. If these mechanisms are sufficiently strong to reduce gene flow between adapted and maladapted forms, trait divergence and ultimately reproductive isolation between populations may be observed (Figure 1(a)). An alternative scenario is depicted in Figure 1(b), where maladapted forms are facilitated by neighboring vegetation, that is, benefactors allow establishment, growth, and reproductive success of 
nonoptimal genotypes. In this case, facilitation may cause allele frequencies in the sink (marginal) to be similar to those of the source (core) habitat. The result would be stable coexistence between two genotypes, a core and a marginal genotype, and thus an overall increased genetic diversity and decreased inbreeding but also greater genetic swamping, that is, a decreased potential to adapt to the marginal conditions. Facilitation may thus be a cohesive force that tends to maintain gene flow between these populations and counteracts diversifying selection. To our knowledge, only one experimental field study has tested this hypothesis and supported our conjecture. This study was conducted on the annual grass Brachypodium distachyon in the Middle East. $B$. distachyon has a broad distribution range and occurs from Mediterranean (core habitat) to semiarid habitats (marginal habitat). Ecotypic differentiation has been observed in this species, with the semiarid ecotypes being more stress-tolerant $[44,52,53]$ and the Mediterranean ecotype having a better ability to cope with competition [44]. When both ecotypes where transplanted to the semiarid environment, both were facilitated by the presence of shrubs but the Mediterranean ecotype could only survive to reproduction underneath the nurse [13]. Facilitation, therefore, enabled a maladapted morph to persist and reproduce in the marginal habitat as described above.

Another scenario would consider the secondary contact of two populations that exhibit trait differentiation. Reinforcement, that is, selection against hybridization, and introgression are two opposing forces that operate during this secondary contact phase, the former enhancing genetic divergence and the latter having the potential to erase it [23]. Secondary contact zones often represent marginal habitats for incipient species (e.g., [54]). Facilitation could favor the cooccurrence of ecotypes according to the mechanism described above and ultimately lead to increased gene flow between ecotypes. Facilitation may also circumvent postzygotic barriers by increasing the fitness of introgressed forms. By increasing the chance of introgression and by preventing reinforcement, we suggest that facilitation could strongly impede further genetic differentiation between ecotypes.

Persistence of core genotypes in marginal habitats increases genetic diversity and thus increases the potential for adapting to changes [46], at least if these occur towards conditions more similar to the core habitat. Higher genetic diversity in marginal habitats compared to core habitats, that is, arid versus Mediterranean environments [55], could support this prediction if this diversity is maintained through time. The trend toward higher genetic variability may be enhanced when the marginal environment varies in time. In that case, the different genotypes inhabiting patches with and without facilitation could have an advantage in different years. Facilitation would then increase the role of temporal variation in supporting stable coexistence of genotypes (e.g., [56]), similar to the storage effect [57] that increases diversity at a species level.

Recent numerical models have been explored under which conditions ecological speciation is likely to occur $[51,58-61]$. The hypothesis that facilitation acts as a cohesive force and increases genetic diversity in marginal habitats can be tested with a spatially and genetically explicit modeling framework derived from Kirkpatrick and Barton [58] and Bridle et al. [62] (Box 1). Our simulations confirm that this modeling framework represents a flexible tool to explore the important set of parameters and scenarios that we believe should be investigated (Box 2). We chose here to explore a reduced set of parameters, that is, size of the patch created by the benefactors and the environmental difference between the core and the marginal habitat (Box 1). Our simulations show that, by producing a mosaic of mild conditions in a harsh environment, benefactors increase gene flow between populations from core and marginal habitats [63], that is, lower $\mathrm{F}_{\mathrm{ST}}$ between populations for both neutral [64] and selected loci (Figures 2(a) and 2(b), resp.). They thus ultimately prevent local adaptation in the harsh environment, as expressed in lower fitness in the marginal environment with facilitation compared to a scenario without facilitation (see Figure 2(c) comparing without benefactors versus smaller patch size benefactors).

\section{Benefactors and Disruptive Selection}

Though facilitation is likely to be a cohesive force, we would also like to touch upon possible conditions under which positive interactions may contribute to, rather than impede, ecological speciation. This is most easily illustrated with particularly stressful environments, where benefactors such as cushion plants in alpine ecosystems or shrubs in waterlimited ecosystems [16], represent islands of milder conditions or islands of fertility. At the community scale, this results in a mosaic of microhabitats determined by the distribution and abundance of benefactors. Such systems have provided the most conclusive evidence for facilitation [65], even if the outcome of biotic interactions may strongly depend upon the particular location of the focal species within this mosaic. Arguably, the patchy distribution of biotically engineered favorable microhabitats within a harsher matrix (e.g., shrubs creating islands of fertility in deserts, see Figure 3) may represent a case at hand for disruptive selection between adjacent subpopulations (see [66] for the example of Bromus erectus adapting to different Thymus vulgaris chemotypes in calcareous grasslands). The benefactors could promote local adaptation in the understory habitats as observed in our simulation when they were forming large patches (Figure 2). Therefore, facilitation could also pave the way to ecological speciation, particularly if directional selection leads to differences in traits that affect the reproductive system, such as flowering time [67]. While this scenario is certainly a possibility, we suggest that it is less likely than the opposite case due to the small spatial scale of environmental heterogeneity usually created by benefactors. Namely, the size of these islands of mild conditions roughly corresponds to the size of the nurse's crown or to a clump of shrubs and is usually small compared to the dispersal kernel and pollination distance [68]. Furthermore, in extreme habitats the nurses also function as a sink for seeds [69]. As a result, it is unlikely that gene flow will be severely restricted between populations of the understory habitats and those of the harsher matrix. Nonetheless, further empirical and 


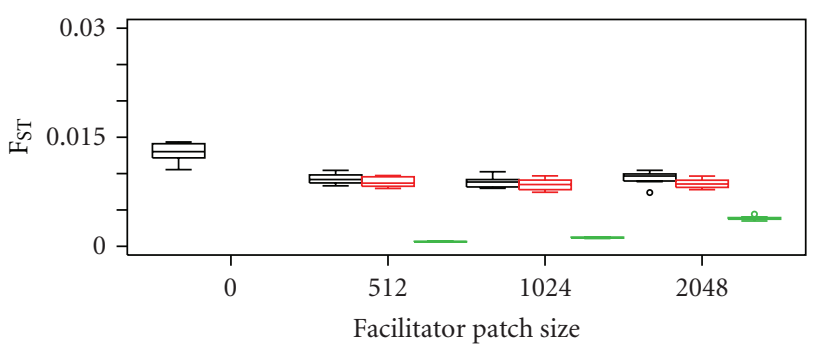

- Core versus marginal

- Benefactor versus core

- Benefactor versus marginal

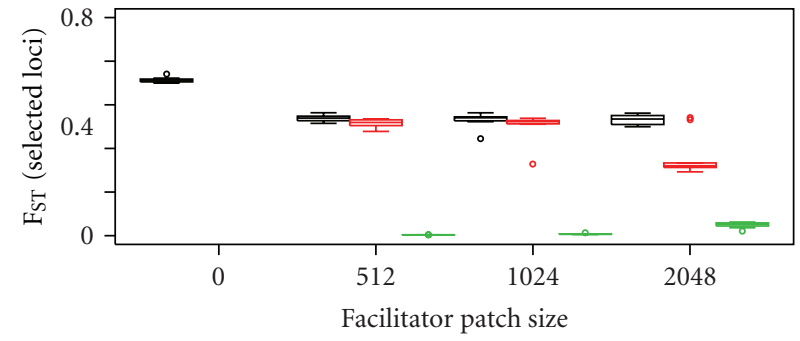

- Core versus marginal

- Benefactor versus core

- Benefactor versus marginal

(a)

(b)

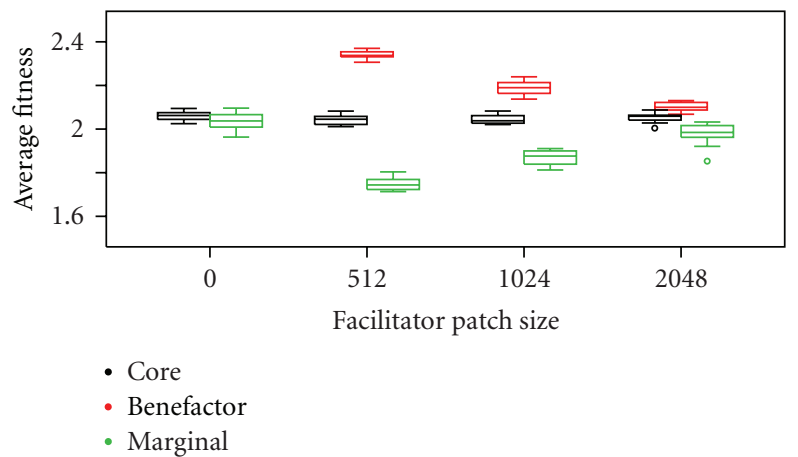

(c)

FIGURE 2: Facilitation acting as a cohesive force and reducing the chance of ecological speciation. (a) represents the population divergence at neutral loci as a function of the size of patch. (b) represents the same population divergence at selected loci. (c) represents the average fitness in each environment as a function of the patch size (Box 1). Consistent with our hypothesis, in the absence of benefactors (i.e., no facilitation, patch size of 0 ), populations were locally adapted and gene flow was reduced between the core and the marginal habitat (higher $\mathrm{F}_{\mathrm{ST}}$ on both neutral and selected loci). In the presence of benefactors, adaptation to the marginal environment was reduced by the increased gene flow from the core population and especially from populations occupying the mild patches created by the benefactors. Individuals occupying these benefactor patches have an increased fitness because of the low population density in the neighboring maladapted marginal environment that inflates the logistic growth part of the fitness function. As the size of the patches increased, migration and gene flow decreased between the patches created by the benefactors and marginal environment (increased $\mathrm{F}_{\mathrm{ST}}$ on both neutral and selected loci). Because of this reduction in gene flow, the average fitness in the marginal environment increased, and consequently the fitness advantage in the benefactor patches decreased. Additionally, the $\mathrm{F}_{\mathrm{ST}}$ for selected loci between the core and the benefactors, even though still high, is reduced at large patch size. This is because each patch has a subpopulation that can achieve the same phenotype using different genotypic combinations. Thus, as the patch size increased the number of patches decreased, the number of subpopulation decreased, the genotypic variance within an environment decreased, and so did the $\mathrm{F}_{\mathrm{ST}}$.

theoretical studies (Box 2) are needed to test for the overwhelming importance of facilitation as a cohesive, rather than a disruptive, force under these particular environmental conditions.

In the context of colonization of a new habitat, where individuals from the population of the core habitat could not persist away from a benefactor in the first place (Figure 4), benefactors could first promote local adaptation at the niche margin and then in the harsh matrix. Recent studies have pointed out the role of intermediate quality habitats in ecotypic differentiation and in the colonisation of a new niche [70]. In the Littorina saxatilis case study, it may be suggested that facilitation played a role in this process given the patchy distribution of mussels and barnacles (in intermediate habitats) associated with the two ecotypes observed in Spain for these species (SU and RB, resp.). However, this hypothesis needs to be properly tested. Thus, on the one hand, positive interactions in intermediate habitats may favor niche broadening and local adaptation. On the other hand, facilitation may contribute to gene flow maintenance between the core and the newly colonized habitats as described in Figure 2. To our opinion, this model system provides excellent opportunities to examine how the spatial and temporal dynamics of positive interactions could interfere with ecological speciation.

\section{Facilitation and Niche Conservatism}

Linkage between facilitation and macroevolutionary processes has recently arisen from experimental work on semiarid Mexican plant communities $[11,71]$. Here, benefactor species were phylogenetically unrelated to beneficiary species; the former belonged to recent Quaternary lineages and were well adapted to the present dry climate, the latter 


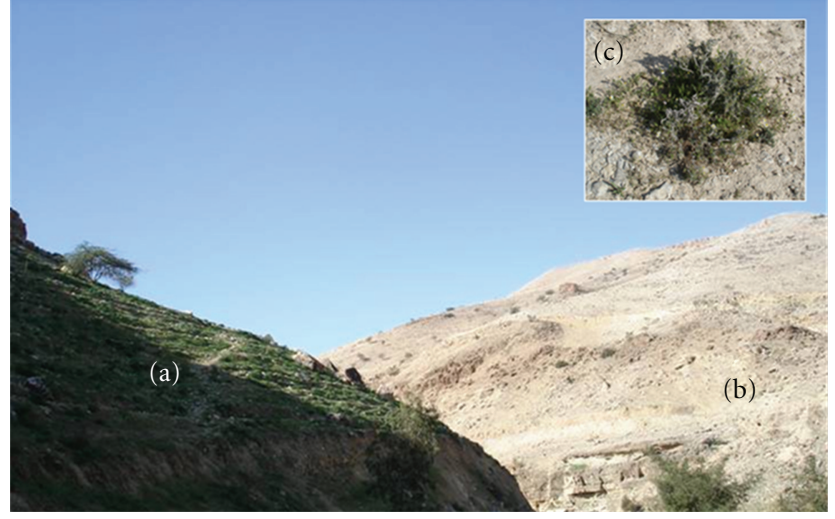

Figure 3: View of a North- (a) and a South- (b) facing slope of Wadi Shuayb, Jordan. The North-facing slope corresponds to a core habitat for many annual (low stature) species and the South-facing slope to a mosaic of marginal habitats with patches of mild habitats created by benefactors (Box 1). (c) Facilitation by the benefactor Gymnocarpos decanter of annual vegetation on the South-facing slope.

showed closer affinities with Tertiary lineages and were drought intolerant [11]. It was hypothesized that less adapted "Tertiary" species survive because of facilitation [11]. In this scenario, our model of facilitation acting as a cohesive force is likely to explain niche conservatism of Tertiary species and the fact that they did not become more stress-tolerant or go extinct through evolutionary time. Transposed to the microevolutionary scale, these results suggest that facilitation may cause a genotype's niche to remain unchanged over time despite environmental changes (Figure 1(c)). Namely, one may imagine that core and marginal habitats are temporally segregated, that is, a population experiences increasingly stressful conditions with time and adaptation to the new conditions is hampered by the persistence of facilitated and maladapted genotypes from more favorable times. Recently, there has been a renewed interest in evaluating niche conservatism across lineages [72], in particular by using species distribution models [73]. To our knowledge, none of these models has properly included the balance between positive and negative interactions along gradients to address the underlying mechanisms and the resulting patterns of niche conservatism.

\section{Conclusion}

We have argued that facilitation can be viewed as a cohesive force limiting genetic and phenotypic differentiation in marginal habitats. Facilitation may also explain the lack of adaptive diversification over time in certain lineages. On the other hand, under very specific conditions, facilitation could also operate as a stepping stone and pave the way to ecological speciation. This calls for a new body of empirical or theoretical studies addressing the strength of these two possible effects of facilitations on ecological speciation (Box 2). Our focus was on the population scale to provide the linkage with mechanisms of speciation. We thus complement recent opinions about the role of various types of facilitation on macro-evolutionary processes [74] and about the effects of evolutionary processes on facilitative interactions [17]. So far, there is no empirical study that has simultaneously examined genetic population structure of beneficiaries and facilitation for contrasting genotypes/phenotypes in natural conditions. Therefore, there is an urgent need to design novel studies that more closely associate community ecologists and evolutionary biologists to tackle these questions (Box 2).

To conclude, we suggest that plant ecologists should invest more into studying biotic interactions at the population level. First, shifting from species to populations and from population to genotypes in the design of ecological experiments is essential to examine the role of facilitation in ecological speciation [12,13]. Second, population models should gain more realism by acknowledging the balance between positive and negative interactions along environmental gradients and their underlying ecological mechanisms. This research agenda should help to bridge the gap between community ecology and evolutionary biology. Bruno et al. [36] claimed that "including facilitation in niche theory will challenge some of our most cherished paradigms." Ecological speciation is another paradigm that is worth revisiting by examining the impact of both negative and positive interactions on the evolutionary history of incipient species. Facilitation is certainly challenging our understanding of the origin of new species.

Boxes.

Box 1: Description of the Modeling Framework. The model of Bridle et al. [62] (initially developed to study adaptation on an environmental gradient) was modified as follows to explore the effect of facilitation on ecological speciation.

The Environment. We used a grid of $8192 \times 4096$ cells wrapped on a torus. Without facilitation, the left half is the core habitat with an optimum $\left(U_{x}\right.$ in Bridle et al. [62]) of $\Theta_{C}$ and the right half is the marginal habitat with optimum of $\boldsymbol{\Theta}_{M}$ and reduced carrying capacity $\left(K_{M}=K_{C} / 2\right.$, the carrying capacity if defined on a circle with radius of 50 cells). In the presence of benefactors, the half righthand side of the grid is fragmented in a checkerboard-like pattern with alternating benefactor and marginal regions. The environments produced by the presence of benefactors have an optimum intermediate between the core and the marginal habitat $\left(\boldsymbol{\Theta}_{F}=\left(\boldsymbol{\Theta}_{\mathrm{C}}+\boldsymbol{\Theta}_{M}\right) / 2\right)$ and the same carrying capacity $\left(K_{F}=K_{C}\right)$ as the core environment.

The Individuals. Individuals are diploid with 128 bi-allelic unlinked loci (64 under selection and 64 neutral). Allele values are either zero or one with a symmetric mutation probability between them of $10^{-4}$. The phenotype $(z)$ is the sum of those loci and thus range from zero to 128 . The fitness of males and females is determined by a logistic growth and local adaptation

$$
w=\max \left(2+r_{f}\left(1-\frac{N}{K}\right)-s \frac{(\theta-z)^{2}}{2}, 0\right),
$$




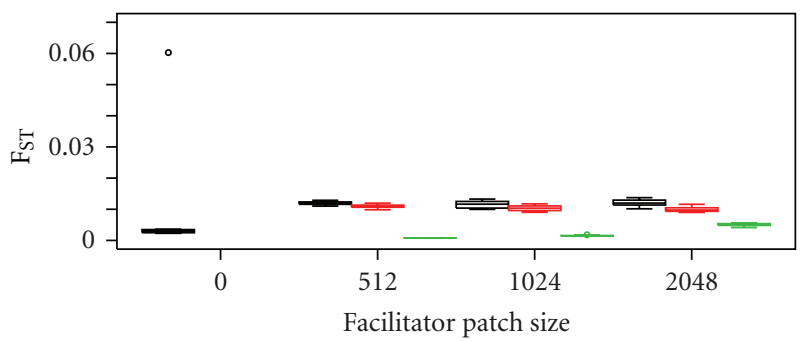

- Core versus marginal

- Benefactor versus core

- Benefactor versus marginal

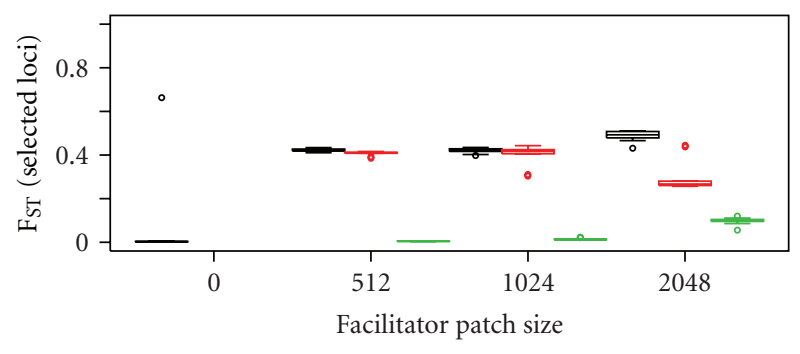

- Core versus marginal

- Benefactor versus core

- Benefactor versus marginal

(a)

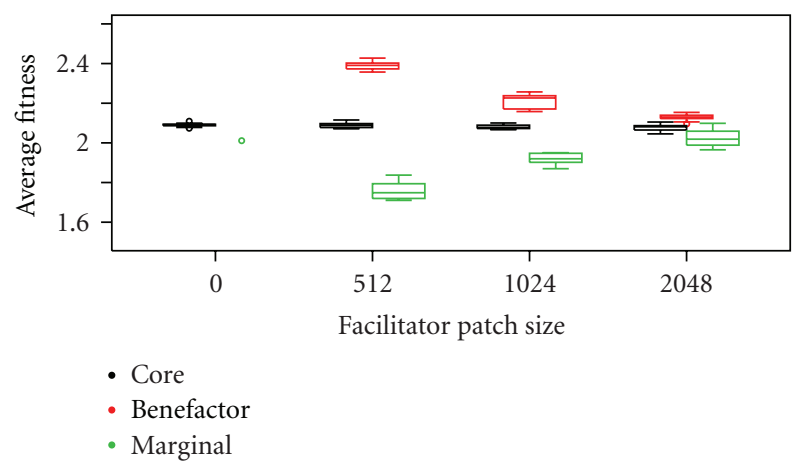

(c)

FIGURE 4: How facilitation may act as a stepping stone for ecological speciation. We looked at that question by increasing the environmental difference between core and marginal habitats. (a) represents the population divergence at neutral loci with either no benefactor or different patch sizes of benefactor in the marginal environment. (b) represents the divergence at selected loci. (c) represents the average fitness in each environment for these two scenarios. The same model and parameters as in Figure 2 were used, except that we increased environmental difference between core and marginal habitats (Box 1). Without benefactor, no population can establish in the marginal environment that is too harsh for migrants to persist. Only dispersers can be observed there, except in one occasion of actual colonization (outlier at patch size of 0 ). In presence of benefactors, all environments are occupied by locally adapted populations and gene flow among environments is reduced. Small patch sizes will increase dispersal between the benefactor and marginal environment as in Figure 2.

where $w$ if the fitness, $r_{f}$ is the maximum rate of increase (set to 0.8 ), $N$ is the local density in a radius of 50 cells, $s$ is the strength of stabilizing selection (set at 0.125 , for the two scenarios presented in Figures 2 and 4 ), and $K$ and $\Theta$ are the carrying capacity and local optimum at the position of the individual. The markers start with no polymorphism (all alleles are zero) and have a mutation rate of $10^{-3}$ that change their value by plus or minus one, so each allele is represented by an integer. We used $\mathrm{F}_{\mathrm{ST}}$ [75] to infer gene flow among populations using neutral markers and selected loci separately.

Reproduction. To find a mate, females scan in a radius of mating distance (MD) set at 150 cells and choose randomly a male with probability proportional to its fitness ( $\left.w_{\text {Male }}\right)$. Each couple leaves an average of $w_{\text {Female }}$ offsprings, drawn from a Poisson distribution. Each offspring is placed at a distance from the mother drawn from a normal distribution of mean zero and standard deviation $D$ (set at 100 cells) in a direction randomly selected from a uniform distribution.

Initial Conditions. We started each simulation with 500 individuals randomly located in the centre $700 \times 700$ of the core environment, and we follow their evolution over 2000 generations. Three patch sizes were used to simulate the presence of benefactors in the harsh environment, smaller patches (512 cells, isolated benefactors in the landscape), intermediate sizes (1024 cells), and large patch sizes (2048 cells). Each parameter set was replicated ten times (the boxplots in Figures 2 and 4 reflect the ten replicates).

Example of Two Contrasting Scenarios. The two sets of simulations that we performed, corresponding to two different scenarios, are presented in Figures 2 and 4. The first scenario presents the case where facilitation inhibits ecological speciation $\left(\boldsymbol{\Theta}_{C}=62, \boldsymbol{\Theta}_{F}=64, \boldsymbol{\Theta}_{M}=66\right.$, Figure 2$)$, and the second scenario presents the case where facilitation promotes ecological speciation $\left(\Theta_{C}=61, \Theta_{F}=64, \Theta_{M}=67\right.$, Figure 4). Following (1), for the first scenario at carrying capacity in both environments, $w$, the fitness for an individual locally adapted to the core environment $(z=62)$ is equal to 2 , but the same individual would have a fitness $w$ equal to 1 in the marginal environment, representing the equivalent of a selection coefficient of $50 \%$. This value is consistent with the average measure of local adaptation recently reported for reciprocal transplantation experiments [76]. In the second 
scenario, we set the environmental difference between the core $\left(\boldsymbol{\Theta}_{C}=61\right)$ and the marginal environment $\left(\boldsymbol{\Theta}_{M}=67\right)$ such that individuals locally adapted to the core environment ( $z=61$ ) could not maintain a viable population in the marginal environment.

\section{Box 2: Facilitation and Ecological Speciation: Ways Forward}

Stress Gradients as a Model System. For empirically studying the role of facilitation in ecological speciation, one should focus on target species with populations widely distributed along stress gradients, and for which facilitation and trait divergence in marginal habitats have been documented. Common examples are gradients of water availability in semiarid landscapes or gradients of temperature along altitudinal gradients. Population genetic structure and inferences about gene flow could be evaluated first descriptively by using neutral or selected markers and by QTL analyses [77]. The next step would be to investigate adaptive genetic variation between microhabitats with and without benefactor. These should be accompanied by experiments testing whether genotypes from "favorable" habitats rely on facilitation to persist and reproduce in marginal habitats [13]. These questions can be addressed with the same experimental designs that are carried out at the species level, that is, reciprocal transplants or sowing experiments coupled with neighbor manipulations.

Which Traits to Measure? A particular challenge to exploring the effects of facilitation is to identify adaptive genetic variation in traits (1) that are under disruptive selection pressure along the investigated gradient and (2) for which trait expression strongly depends on the balance between stress tolerance and success in the core habitat. Seed size, for example, is a good candidate because it is related to seedling size and tolerance of hazards for young seedlings [78]. As competitive ability and stress tolerances ability are related to the probability of being facilitated [5], traits linked with the resource capture and utilization, such as leaf traits, plant stature traits, and root traits, need to be considered as well (see [79]).

A key trait would be both under strong selective pressure and closely related to mate choice, the so-called "magic traits" $[45,51,80]$. Because the benefactor may modify the length of the favorable season, for instance, phenological traits such as time to flowering or length of the life cycle $[44,81]$ may fall in this category and are worth examining. Depending on how benefactors affect these key traits, facilitation could potentially either hinder, accelerate or even lead to ecological speciation (see "Section 4").

Theory and Simulations. There are large open avenues for tackling these questions with spatially explicit models of population dynamics, building on a rich set of various modeling frameworks $[26,51,62,70,82-84]$. We present here an example (Figures 2 and 4 ) but such models should be refined to explore the specific feature of facilitation and its implication on adaptive diversification along gradients and other spatiotemporal configurations. Namely, the crucial role of dispersal for adaptation to marginal habitats should be investigated in the presence of facilitation. For example, an important consequence of facilitation may be that it enhances "effective" dispersal from the source to sinks, because establishment probabilities of maladapted genotypes are enhanced. Furthermore, the sensitivity of model outputs to parameters controlling (i) the shape of the competition versus facilitation balance along ecological gradients, that is still widely debated $[6,31]$, (ii) the spatial distribution and dynamics of nurse plants in marginal habitats, (iii) the complex interactions among benefactors and beneficiaries [17], and (iv) the dispersal ability and life history traits of focal species, has to be investigated. Ultimately, these models should help us sharpen and phrase testable hypotheses about the relative importance of facilitation on adaptation and ecological speciation. Finally, a spatially and genetically explicit model [51, 59-61] could couple both benefactor effect on beneficiaries' microevolutionary responses (this study) and the feedback effect of beneficiaries on population and evolutionary dynamics of benefactor species (cost of facilitation, [17]). Such an approach would shed light on how the evolutionary consequence of facilitation affects community assembly and dynamics.

\section{Authors' Contribution}

P. Liancourt wrote the first draft of the manuscript, X. Thibert-Plante performed the modelling work. All authors made a substantial intellectual contribution to the paper and its revision. P. Choler, N. Gross and X. Thibert-Plante had equal contribution to the paper.

\section{Acknowledgment}

P. Liancourt and K. Tielbörger were supported by the GLOWA Jordan River Project funded by the German Ministry of Science and Education (BMBF), and further support was granted to K. Tielbörger by the German Research Foundation (TI338/10-1). P. Choler was partly funded by a Marie-Curie fellowship (contract CASOAR MOIF-CT-2006039688). X. Thibert-Plante conducted this work while a Postdoctoral Fellow at the National Institute for Mathematical and Biological Synthesis, an institute sponsored by the National Science Foundation, the U.S. Department of Homeland Security, and the U.S. Department of Agriculture through NSF Award no. EF-0832858, with additional support from The University of Tennessee, Knoxville, and Le Fonds Québécois de la Recherche sur la Nature et les Technologies (FQRNT) directly to X. Thibert-Plante. This research was also partially supported by NIH Grant GM56693 to S. Gavrilets. The authors thank R. Glade for editing the paper to improve the English, J. Bridle and J. Polechová for sharing with them details of their model, S. Gavrilets, A. P. Henry, R. M. Callaway, and R. Michalet for feedback on the paper, and R. Faria and the anonymous reviewers for helpful comments on the paper and suggesting the analysis of the fixation index on the selected loci. 


\section{References}

[1] M. D. Bertness and R. Callaway, "Positive interactions in communities," Trends in Ecology and Evolution, vol. 9, no. 5, pp. 191-193, 1994.

[2] R. W. Brooker, F. T. Maestre, R. M. Callaway et al., "Facilitation in plant communities: the past, the present, and the future," Journal of Ecology, vol. 96, no. 1, pp. 18-34, 2008.

[3] T. Day and K. A. Young, "Competitive and facilitative evolutionary diversification,” BioScience, vol. 54, no. 2, pp. 101-109, 2004.

[4] P. Choler, B. Erschbamer, A. Tribsch, L. Gielly, and P. Taberlet, "Genetic introgression as a potential to widen a species' niche: insights from alpine Carex curvula," Proceedings of the National Academy of Sciences of the United States of America, vol. 101, no. 1, pp. 171-176, 2004.

[5] P. Liancourt, R. M. Callaway, and R. Michalet, "Stress tolerance and competitive-response ability determine the outcome of biotic interactions," Ecology, vol. 86, no. 6, pp. 1611-1618, 2005.

[6] F. T. Maestre, R. M. Callaway, F. Valladares, and C. J. Lortie, "Refining the stress-gradient hypothesis for competition and facilitation in plant communities," Journal of Ecology, vol. 97, no. 2, pp. 199-205, 2009.

[7] C. Darwin, On the Origin of Species by Means of Natural Selection, or the Preservation of Favoured Races in the Struggle for Life, John Murray, London, UK, 1859.

[8] M. L. Rosenzweig, “Competitive speciation,” Biological Journal of the Linnean Society, vol. 10, no. 3, pp. 275-289, 1978.

[9] D. Schluter, "Experimental evidence that competition promotes divergence in adaptive radiation," Science, vol. 266, no. 5186, pp. 798-801, 1994.

[10] D. I. Bolnick, "Can intraspecific competition drive disruptive selection? An experimental test in natural populations of sticklebacks," Evolution, vol. 58, no. 3, pp. 608-618, 2004.

[11] A. Valiente-Banuet, A. V. Rumebe, M. Verdú, and R. M. Callaway, "Modern quaternary plant lineages promote diversity through facilitation of ancient tertiary lineages," Proceedings of the National Academy of Sciences of the United States of America, vol. 103, no. 45, pp. 16812-16817, 2006.

[12] E. K. Espeland and K. J. Rice, "Facilitation across stress gradients: the importance of local adaptation," Ecology, vol. 88, no. 9, pp. 2404-2409, 2007.

[13] P. Liancourt and K. Tielbörger, "Ecotypic differentiation determines the outcome of positive interactions in a dryland annual plant species," Perspectives in Plant Ecology, Evolution and Systematics, vol. 13, no. 4, pp. 259-264, 2011.

[14] J. H. Vandermeer and D. H. Boucher, "Varieties of mutualistic interaction in population models," Journal of Theoretical Biology, vol. 74, no. 4, pp. 549-558, 1978.

[15] J. L. Bronstein, "The evolution of facilitation and mutualism," Journal of Ecology, vol. 97, no. 6, pp. 1160-1170, 2009.

[16] R. M. Callaway, Positive Interactions and Interdependence in Plant Communities, Springer, Dordrecht, The Netherlands, 2007.

[17] R. Michalet, S. Xiao, B. Touzard et al., "Phenotypic variation in nurse traits and community feedbacks define an alpine community," Ecology Letters, vol. 14, no. 5, pp. 433-443, 2011.

[18] K. Tielbörger and R. Kadmon, "Temporal environmental variation tips the balance between facilitation and interference in desert plants," Ecology, vol. 81, no. 6, pp. 1544-1553, 2000.

[19] M. T. J. Johnson and J. R. Stinchcombe, "An emerging synthesis between community ecology and evolutionary biology,"
Trends in Ecology and Evolution, vol. 22, no. 5, pp. 250-257, 2007.

[20] J. R. Haloin and S. Y. Strauss, "Interplay between ecological communities and evolution: review of feedbacks from microevolutionary to macroevolutionary scales," Annals of the New York Academy of Sciences, vol. 1133, pp. 87-125, 2008.

[21] D. M. Post and E. P. Palkovacs, "Eco-evolutionary feedbacks in community and ecosystem ecology: interactions between the ecological theatre and the evolutionary play," Philosophical Transactions of the Royal Society B, vol. 364, no. 1523, pp. 1629-1640, 2009.

[22] T. W. Schoener, "The newest synthesis: understanding the interplay of evolutionary and ecological dynamics," Science, vol. 331, no. 6016, pp. 426-429, 2011.

[23] D. Schluter, "Ecology and the origin of species," Trends in Ecology and Evolution, vol. 16, no. 7, pp. 372-380, 2001.

[24] J. S. McKinnon, S. Mori, B. K. Blackman et al., "Evidence for ecology's role in speciation," Nature, vol. 429, no. 6989, pp. 294-298, 2004.

[25] H. D. Rundle and P. Nosil, "Ecological speciation," Ecology Letters, vol. 8, no. 3, pp. 336-352, 2005.

[26] M. Doebeli and U. Dieckmann, "Speciation along environmental gradients," Nature, vol. 421, no. 6920, pp. 259-264, 2003.

[27] C. C. Wilmers, R. L. Crabtree, D. W. Smith, K. M. Murphy, and W. M. Getz, "Trophic facilitation by introduced top predators: grey wolf subsidies to scavengers in Yellowstone National Park," Journal of Animal Ecology, vol. 72, no. 6, pp. 909-916, 2003.

[28] F. Bulleri, "Facilitation research in marine systems: state of the art, emerging patterns and insights for future developments," Journal of Ecology, vol. 97, no. 6, pp. 1121-1130, 2009.

[29] M. G. A. Van Der Heijden and T. R. Horton, "Socialism in soil? the importance of mycorrhizal fungal networks for facilitation in natural ecosystems," Journal of Ecology, vol. 97, no. 6, pp. 1139-1150, 2009.

[30] D. J. Hodgson, R. B. Hitchman, A. J. Vanbergen, R. S. Hails, R. D. Possee, and J. S. Cory, "Host ecology determines the relative fitness of virus genotypes in mixed-genotype nucleopolyhedrovirus infections," Journal of Evolutionary Biology, vol. 17, no. 5, pp. 1018-1025, 2004.

[31] R. Michalet, R. W. Brooker, L. A. Cavieres et al., "Do biotic interactions shape both sides of the humped-back model of species richness in plant communities?" Ecology Letters, vol. 9, no. 7, pp. 767-773, 2006.

[32] S. D. Hacker and S. D. Gaines, "Some implications of direct positive interactions for community species diversity," Ecology, vol. 78, no. 7, pp. 1990-2003, 1997.

[33] R. M. Callaway, R. W. Brooker, P. Choler et al., "Positive interactions among alpine plants increase with stress," Nature, vol. 417, no. 6891, pp. 844-848, 2002.

[34] L. A. Cavieres and E. I. Badano, "Do facilitative interactions increase species richness at the entire community level?" Journal of Ecology, vol. 97, no. 6, pp. 1181-1191, 2009.

[35] L. Pellissier, K. Anne Bråthen, J. Pottier et al., "Species distribution models reveal apparent competitive and facilitative effects of a dominant species on the distribution of tundra plants," Ecography, vol. 33, no. 6, pp. 1004-1014, 2010.

[36] J. F. Bruno, J. J. Stachowicz, and M. D. Bertness, "Inclusion of facilitation into ecological theory," Trends in Ecology and Evolution, vol. 18, no. 3, pp. 119-125, 2003.

[37] N. Gross, P. Liancourt, P. Choler, K. N. Suding, and S. Lavorel, "Strain and vegetation effects on local limiting resources explain the outcomes of biotic interactions," Perspectives in 
Plant Ecology, Evolution and Systematics, vol. 12, no. 1, pp. 919, 2010.

[38] M. Abrams, "Genotypic and phenotypic variation as stress adaptations in temperate tree species-a review of several casestudies," Tree Physiology, vol. 14, pp. 833-842, 1994.

[39] G. E. Rehfeldt, C. C. Ying, D. L. Spittlehouse, and D. A. Hamilton, "Genetic responses to climate in Pinus contorta: niche breadth, climate change, and reforestation," Ecological Monographs, vol. 69, no. 3, pp. 375-407, 1999.

[40] M. Ackermann and M. Doebeli, "Evolution of niche width and adaptive diversification," Evolution, vol. 58, no. 12, pp. 25992612, 2004.

[41] M. Slatkin, "Frequency- and density-dependent selection on a quantitative character," Genetics, vol. 93, no. 3, pp. 755-771, 1979.

[42] F. B. Christiansen and V. Loeschcke, "Evolution and intraspecific exploitative competition I. One-locus theory for small additive gene effects," Theoretical Population Biology, vol. 18, no. 3, pp. 297-313, 1980.

[43] T. J. Kawecki, "Adaptation to marginal habitats," Annual Review of Ecology, Evolution, and Systematics, vol. 39, pp. 321342, 2008.

[44] P. Liancourt and K. Tielbörger, "Competition and a short growing season lead to ecotypic differentiation at the two extremes of the ecological range," Functional Ecology, vol. 23, no. 2, pp. 397-404, 2009.

[45] S. Gavrilets, Fitness Landscapes and the Origin of Species, Princeton University Press, 2004.

[46] R. D. Holt and T. H. Keitt, "Species' borders: a unifying theme in ecology," Oikos, vol. 108, no. 1, pp. 3-6, 2005.

[47] T. Lenormand, "Gene flow and the limits to natural selection," Trends in Ecology and Evolution, vol. 17, no. 4, pp. 183-189, 2002.

[48] H. Gonzalo-Turpin and L. Hazard, "Local adaptation occurs along altitudinal gradient despite the existence of gene flow in the alpine plant species Festuca eskia," Journal of Ecology, vol. 97, no. 4, pp. 742-751, 2009.

[49] P. Nosil, T. H. Vines, and D. J. Funk, "Perspective: reproductive isolation caused by natural selection against immigrants from divergent habitats," Evolution, vol. 59, no. 4, pp. 705-719, 2005.

[50] M. R. Servedio and M. A. F. Noor, "The role of reinforcement in speciation: theory and data," Annual Review of Ecology, Evolution, and Systematics, vol. 34, pp. 339-364, 2003.

[51] X. Thibert-Plante and A. P. Hendry, "Five questions on ecological speciation addressed with individual-based simulations," Journal of Evolutionary Biology, vol. 22, no. 1, pp. 109-123, 2009.

[52] J. Aronson, J. Kigel, A. Shmida, and J. Klein, "Adaptive phenology of desert and Mediterranean populations of annual plants grown with and without water stress," Oecologia, vol. 89, no. 1, pp. 17-26, 1992.

[53] J. Aronson, J. Kigel, and A. Shmida, "Reproductive allocation strategies in desert and Mediterranean populations of annual plants grown with and without water stress," Oecologia, vol. 93, no. 3, pp. 336-342, 1993.

[54] P. Choler, R. Michalet, and R. M. Callaway, "Facilitation and competition on gradients in alpine plant communities," Ecology, vol. 82, no. 12, pp. 3295-3308, 2001.

[55] S. Volis, S. Mendlinger, L. Olsvig-Whittaker, U. N. Safriel, and N. Orlovsky, "Phenotypic variation and stress resistance in core and peripheral populations of Hordeum spontaneum," Biodiversity and Conservation, vol. 7, no. 6, pp. 799-813, 1998.
[56] A. S. Jump and J. Peñuelas, "Running to stand still: adaptation and the response of plants to rapid climate change," Ecology Letters, vol. 8, no. 9, pp. 1010-1020, 2005.

[57] A. L. Angert, T. E. Huxman, P. Chesson, and D. L. Venable, "Functional tradeoffs determine species coexistence via the storage effect," Proceedings of the National Academy of Sciences of the United States of America, vol. 106, no. 28, pp. 11641$11645,2009$.

[58] M. Kirkpatrick and N. H. Barton, "Evolution of a species' range," American Naturalist, vol. 150, no. 1, pp. 1-23, 1997.

[59] S. Gavrilets, A. Vose, M. Barluenga, W. Salzburger, and A. Meyer, "Case studies and mathematical models of ecological speciation. 1. Cichlids in a crater lake," Molecular Ecology, vol. 16, no. 14, pp. 2893-2909, 2007.

[60] S. Gavrilets and A. Vose, "Case studies and mathematical models of ecological speciation. 2. Palms on an oceanic island," Molecular Ecology, vol. 16, no. 14, pp. 2910-2921, 2007.

[61] X. Thibert-Plante and A. P. Hendry, "The consequences of phenotypic plasticity for ecological speciation," Journal of Evolutionary Biology, vol. 24, no. 2, pp. 326-342, 2011.

[62] J. R. Bridle, J. Polechová, M. Kawata, and R. K. Butlin, "Why is adaptation prevented at ecological margins? New insights from individual-based simulations," Ecology Letters, vol. 13, no. 4, pp. 485-494, 2010.

[63] J. A. Endler, "Geographic Variation, Speciation, and Clines," Princeton University.

[64] X. Thibert-Plante and A. P. Hendry, "When can ecological speciation be detected with neutral loci?" Molecular Ecology, vol. 19, no. 11, pp. 2301-2314, 2010.

[65] R. Tirado and F. I. Pugnaire, "Shrub spatial aggregation and consequences for reproductive success," Oecologia, vol. 136, no. 2, pp. 296-301, 2003.

[66] B. K. Ehlers and J. Thompson, "Do co-occurring plant species adapt to one another? The response of Bromus erectus to the presence of different Thymus vulgaris chemotypes," Oecologia, vol. 141, no. 3, pp. 511-518, 2004.

[67] V. Savolainen, M. C. Anstett, C. Lexer et al., "Sympatric speciation in palms on an oceanic island," Nature, vol. 441, no. 7090, pp. 210-213, 2006.

[68] A. Gros, H. Joachim Poethke, and T. Hovestadt, "Evolution of local adaptations in dispersal strategies," Oikos, vol. 114, no. 3 , pp. 544-552, 2006.

[69] J. Flores and E. Jurado, "Are nurse-protege interactions more common among plants from arid environments?" Journal of Vegetation Science, vol. 14, no. 6, pp. 911-916, 2003.

[70] S. Sadedin, J. Hollander, M. Panova, K. Johannesson, and S. Gavrilets, "Case studies and mathematical models of ecological speciation. 3: ecotype formation in a Swedish snail," Molecular Ecology, vol. 18, no. 19, pp. 4006-4023, 2009.

[71] A. Valiente-Banuet and M. Verdú, "Facilitation can increase the phylogenetic diversity of plant communities," Ecology Letters, vol. 10, no. 11, pp. 1029-1036, 2007.

[72] J. J. Wiens and C. H. Graham, "Niche conservatism: integrating evolution, ecology, and conservation biology," Annual Review of Ecology, Evolution, and Systematics, vol. 36, pp. 519539, 2005.

[73] P. B. Pearman, A. Guisan, O. Broennimann, and C. F. Randin, "Niche dynamics in space and time," Trends in Ecology and Evolution, vol. 23, no. 3, pp. 149-158, 2008.

[74] Z. Kikvidze and R. M. Callaway, "Ecological facilitation may drive major evolutionary transitions," BioScience, vol. 59, no. 5, pp. 399-404, 2009.

[75] B. S. Weir, Genetic Data Analysis II: Methods for Discrete Population Genetic Data, Sinauer Associates, Sunderland, Mass, USA, 1996. 
[76] J. Hereford, "A quantitative survey of local adaptation and fitness trade-offs," American Naturalist, vol. 173, no. 5, pp. 579-588, 2009.

[77] P. Nosil, D. J. Funk, and D. Ortiz-Barrientos, "Divergent selection and heterogeneous genomic divergence," Molecular Ecology, vol. 18, no. 3, pp. 375-402, 2009.

[78] A. T. Moles and M. Westoby, "Seedling survival and seed size: a synthesis of the literature," Journal of Ecology, vol. 92, no. 3, pp. 372-383, 2004.

[79] V. Maire, N. Gross, L. Da Silveira Pontes, C. Picon-Cochard, and J. F. Soussana, "Trade-off between root nitrogen acquisition and shoot nitrogen utilization across 13 co-occurring pasture grass species," Functional Ecology, vol. 23, no. 4, pp. 668-679, 2009.

[80] M. R. Servedio, G. S.V. Doorn, M. Kopp, A. M. Frame, and P. Nosil, "Magic traits in speciation: "magic" but not rare?" Trends in Ecology and Evolution, vol. 26, no. 8, pp. 389-397, 2011.

[81] M. Petrů, K. Tielbörger, R. Belkin, M. Sternberg, and F. Jeltsch, "Life history variation in an annual plant under two opposing environmental constraints along an aridity gradient," Ecography, vol. 29, no. 1, pp. 66-74, 2006.

[82] A. Hastings and S. Harrison, "Metapopulation dynamics and genetics," Annual Review of Ecology and Systematics, vol. 25, pp. 167-188, 1994.

[83] J. Molofsky and J. D. Bever, "A new kind of ecology?" BioScience, vol. 54, no. 5, pp. 440-446, 2004.

[84] J. M. J. Travis, R. W. Brooker, E. J. Clark, and C. Dytham, "The distribution of positive and negative species interactions across environmental gradients on a dual-lattice model," Journal of Theoretical Biology, vol. 241, no. 4, pp. 896-902, 2006. 

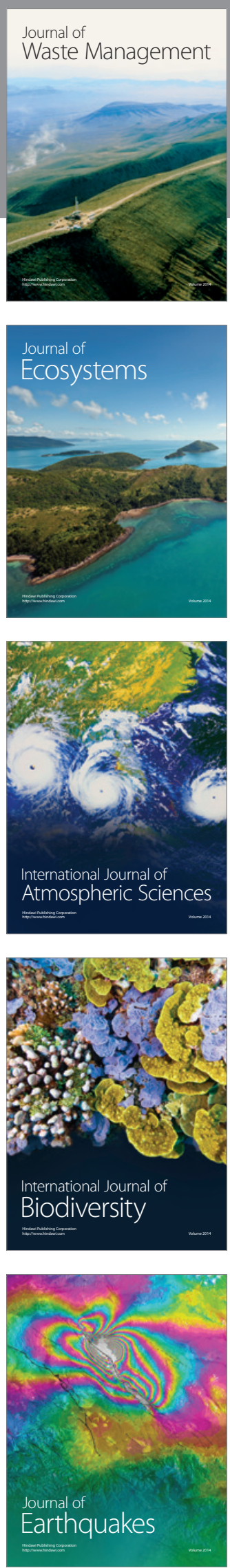
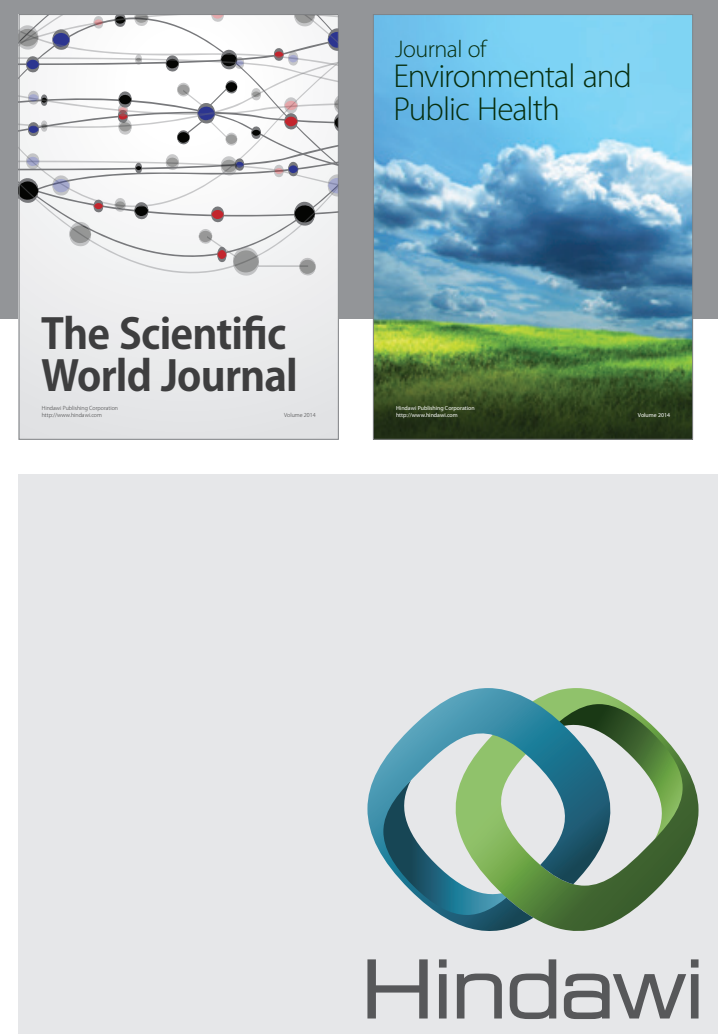

Submit your manuscripts at

http://www.hindawi.com
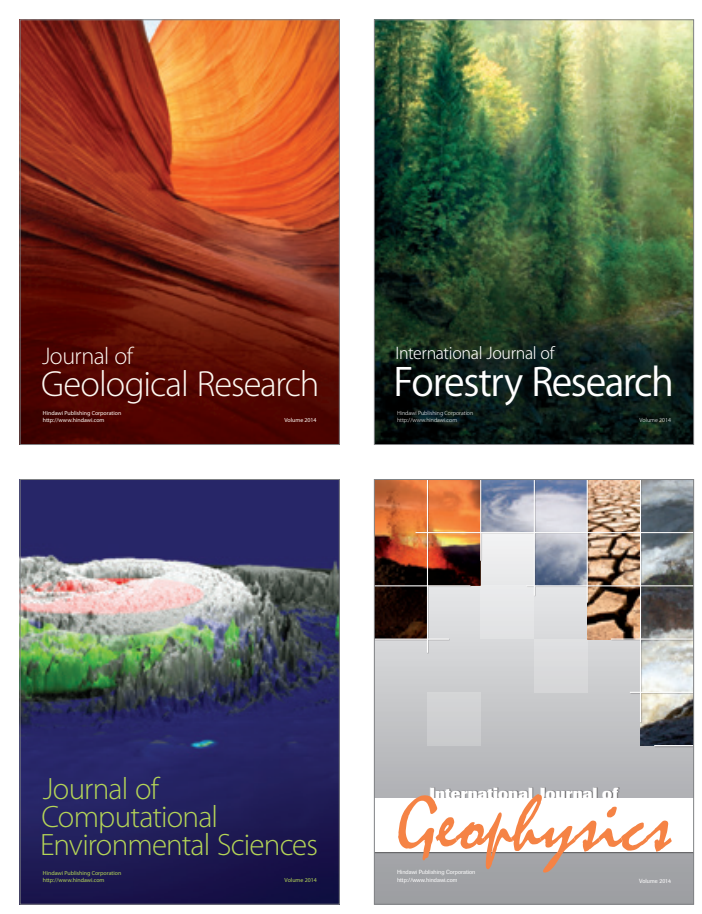
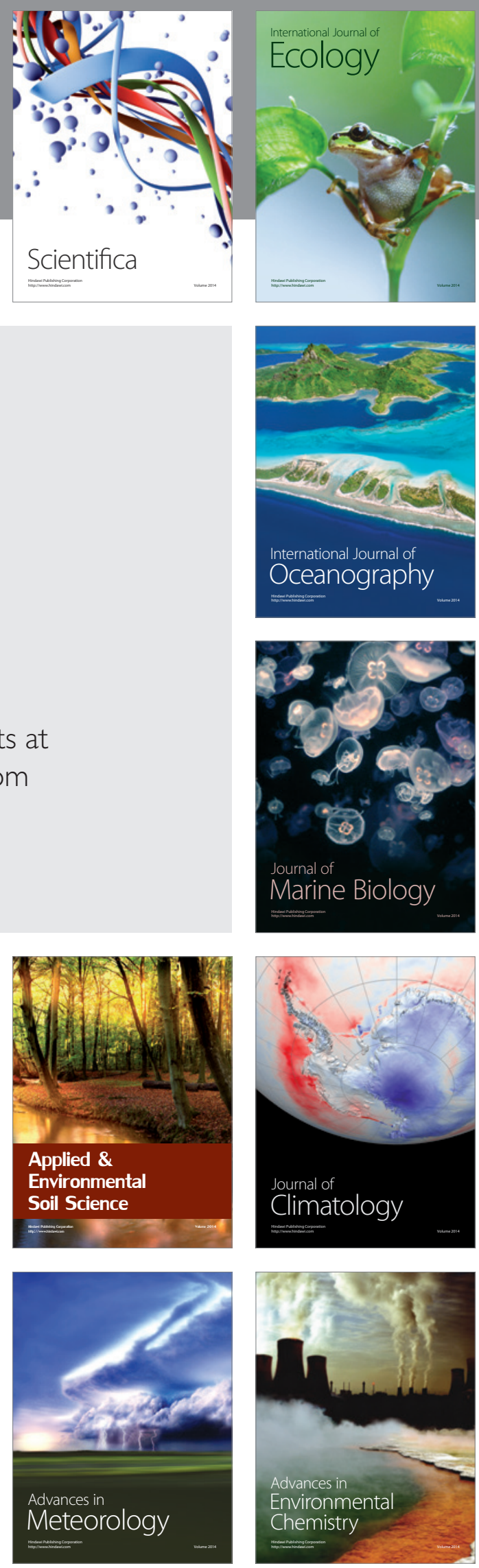